\author{
ANNALES \\ POLONICI MATHEMATICI \\ XXVIII (1973)
}

\title{
On new definitions and interpretations of union, hyperasymptotic and hypernormal curves in the three-dimensional Euclidean space
}

\author{
by H.K.N. Trivedi and S. C. RASTOGI (Lueknow)
}

1. Introduction. Let $\hat{\lambda}, \hat{\omega}_{1}$ and $\hat{\omega}_{2}$ be, respectively, the unit vector along a line of the rectilinear congruence, the principal normal unit vector of $\hat{\lambda}$ relative to a curve $C: \underline{x}=\underline{x}(s)$ on the surface of reference $S: \underline{x}=\underline{x}\left(u^{a}\right)$ $(\alpha=1,2)$ and the binormal unit vector of $\hat{\lambda}$ relative to $C$. In two of our earlier papers [4] and [7] we have considered the planes spanned by the pairs of vectors $\left(\hat{\lambda}, \hat{\omega}_{1}\right)$ and $\left(\hat{\lambda}, \hat{\omega}_{2}\right)$ which have been called the intrinsic oseulating plane and the relative rectifying plane, respectively. In the cases when the tangent, the principal normal or the binormal to the curve $C$, lie in these planes, we have called the curve in question an $a-, \beta-, \gamma$-and $T_{\lambda^{-}}, N_{\lambda^{-}}, B_{\lambda^{-}}$curve, respectively. In this paper we define the relative normal plane as the plane spanned by $\hat{\omega}_{1}$ and $\hat{\omega}_{2}$. In the cases when the tangent, the principal normal or the binormal to the curve $C$, lie respectively in this plane we define and study three types of curves. Incidentally, the results show that these curves are not new but the well-known curves, viz., hypernormal curves [1], hyperasymptotic curves [2] and union curves [5] respectively. Thus in this paper we give new definitions to the known curves. We study some properties of these curves by obtaining the expressions for the curvature and torsion of these curves.

Throughout this paper we shall assume that the functions involved are continuous and differentiable up to the required order.

2. Prerequisites. At any point $P(\underline{x})$ of $S$, the unit vector $\hat{\lambda}$ may be expressed as

$$
\hat{\lambda}=p^{a} \underline{x}_{,}+q \hat{N},
$$

where $p^{\alpha}$ are the contravariant components of a vector in $S, x_{, \alpha}$ is used for $\partial \underline{x} / \partial u^{a}, \hat{N}\left(=\underline{x}_{1} \times \underline{x}, 2 / \mid \underline{x}, 1 \times \underline{q}, 2\right)$ stands for the unit normal to $S$ at $P$ and $q$ is a $\bar{W}$-scalar $\left(q^{\prime}=\operatorname{sgn}\left|\partial u^{a^{\prime}}\right| \partial u^{a} \mid q\right) . \underline{x}_{, a}$ may also be regarded as the covariant derivative of $x$ with respect to $u^{a}$ based on the first fundamental tensor $g_{a \beta}=\underline{x}_{,} \cdot \underline{x}_{, \beta}$ of $S$. 
Differentiating (2.1) with respect to $s$ and applying the Gauss and Weingarten equations

$$
\underline{x}_{, a \beta}=d_{a \beta} \hat{N} \quad \text { and } \quad \hat{N}_{, \beta}=-d_{\alpha \gamma} g^{\gamma \delta} \underline{x}_{, \delta}
$$

Wve get

$$
d \hat{\lambda} / d s=\left(\mu_{\beta}^{a} \underline{x}, a+v_{\beta} \hat{N}\right) u^{\prime \beta}
$$

where

$$
\mu_{\beta}^{a} \equiv p_{, \beta}^{a}-q d_{\beta \delta} g^{\delta a}
$$

and

$$
\nu_{\beta} \equiv q_{, \beta}+p^{\alpha} d_{\alpha \beta},
$$

$d_{a \beta}$ being the second fundamental tensor of $S$.

Nirmala [3] has defined Frenet formulae for the vector field $\hat{\lambda}$, with respect to a curve $C$ in a Riemannian space, which, in particular, for the three-dimensional Euclideain space read as follows:

$$
\begin{aligned}
d \cdot \hat{\lambda} / d s & =K_{1} \hat{\omega}_{1}, \\
d \hat{\omega}_{1} / d s & =K_{2} \hat{\omega}_{2}-K_{1} \hat{\lambda} \\
d \hat{\omega}_{2} / d s & =-K_{2} \hat{\omega}_{1},
\end{aligned}
$$

where $K_{1}$ is the absolute curvature of the congruence and $K_{2}$ is the absolute torsion of the congruence with respect to the curve $C$.

3. Union curves. We define the relative normal plane as the plane spanned by the vectors $\hat{\omega}_{1}$ and $\hat{\omega}_{2}$.

A curve $C$ is said to be anion curve if its binormal lies in the relative normal plane.

Thus, by definition, we have for a union curve

$$
\left(d \underline{x} / d s \times d^{2} \underline{x} / d s^{2}\right) \cdot\left(\hat{\omega}_{1} \times \hat{\omega}_{2}\right)=0
$$

where $d \underline{x} / d s$ is the unit tangent vector to $C$ and $d^{2} \underline{x} / d s^{2}$ gives the direction of the principal normal to $C$. Since

$$
\begin{aligned}
\hat{\omega}_{1} \times \hat{\omega}_{2} & =\hat{\lambda}, \\
d \underline{x} / d s & =\underline{x}_{, a}, u^{\prime a}
\end{aligned}
$$

and

$$
d^{2} \underline{x} / d s^{2}=\underline{x}_{, u} p^{u}+k_{n} \hat{N}
$$


therefore equation (3.1) after simplification leads to

$$
e_{\mathrm{a \beta}} u^{\prime \alpha}\left(q \varrho^{\beta}-k_{n} p^{\beta}\right)=0,
$$

which is the equation of a union curve [5], where $e_{\alpha \beta}=x_{\alpha} \times x_{, \beta} \cdot \hat{N}$.

To obtain the alternative form we express $d \underline{x} / d s \times d^{2} \underline{x} / d s^{2}$ as follows:

$$
(d \underline{x} / d s) \times\left(d^{2} \underline{x} / d s^{2}\right)=K_{b}\left(\hat{\omega}_{1} \cos \psi+\hat{\omega}_{2} \sin \psi\right),
$$

where $\Pi_{b}$ is the curvature of union curves and $\psi$ is the angle between the binormal to the curve $C$ and $\hat{\omega}_{1}$.

Multiplying equation (3.4) by $\hat{\omega}_{1}$ and $\hat{\omega}_{2}$; respectively, in the sense of inner product and applying equations (2.1), (2.2), (2.4), (3.2a) and (3.2b) we obtain after simplification

$$
K_{1} K_{\iota} \cos \psi=e_{a \beta} u^{\prime a} u^{\prime \beta}\left(\varrho^{\beta} \nu_{\delta}-k_{n} \mu_{\delta}^{\beta}\right)
$$

and

$$
K_{1} K_{b} \sin \psi=p_{a} u^{\prime 2}\left(\mu_{\beta \delta} e^{\beta}+\nu_{\delta} k_{n}\right) u^{\prime \delta}-k_{c} \mu_{\beta \delta} u^{\prime \beta} u^{\prime \delta}
$$

where [2]:

$$
\mu_{\beta \delta}=g_{a \beta} \mu_{\delta}^{a}
$$

and

$$
k_{s}=\varrho^{a} p_{a}+q k_{n} .
$$

Squaring equatious (3.5a, b) and adding we get

$$
\begin{aligned}
& K_{1}^{2} K_{b}^{2}=\left\{e_{\alpha \beta} u^{\prime a} u^{\prime \delta}\left(\varrho^{\beta} v_{\delta}-k_{n} \mu_{\delta}^{\beta}\right)\right\}^{2}+ \\
& +\left\{p_{a} u^{\prime \alpha}\left(\mu_{\beta \delta} \varrho^{\beta}+\nu_{\delta} k_{n}\right) u^{\prime \delta}-k_{c} \mu_{\beta \delta} u^{\prime \beta} u^{\prime \delta}\right\}^{2} .
\end{aligned}
$$

Equation (3.7) is the alternative form of the differential equation of union curves in the three-dimensional Euclidean space.

Since for a $B_{\lambda}$-curve [ $\left.\tau\right]$ we have

$$
e_{\alpha \beta} u^{\prime a} u^{\prime \delta}\left(\varrho^{\beta} v_{\delta}-k_{n} \mu_{\delta}^{\beta}\right)=0 \text {, }
$$

therefore equation (3.5a) leads to $K_{1} K_{l} \cos \psi=0$, which implies

THEOREM (3.1). The necessary and sufficient condition for a union curve to be a $B_{\lambda}$-curve is that it satisfies one of the following conditions:

(i) the absolute curvature of the congruence vanishes,

(ii) the curvature of the union curves vanishes,

(iii) the binormal to the curve $C$ is orthogonal to $\hat{\omega}_{1}$.

Multiplying equation (3.4) by $\dot{N}$ in the sense of inner product and using (2.1), (2.2), (3.2a) and (3.2b) we obtain after suitable calculations

$$
K_{1} k_{0}=K_{b}\left(\nu_{\beta} u^{\prime \beta} \cos \psi+e_{\alpha \beta} p^{a} \mu_{\gamma}^{\beta} u^{\prime \prime} \sin \psi\right),
$$


where

$$
k_{g}=e_{u p} u^{\prime a} Q^{\beta} .
$$

Equations (3.5a, b) and (3.9) are the alternative forms, which represent the curvature of the union curves.

If we denote the unit binormal vector or a union curve by $\hat{b}$, then equation (3.4), when differentiated with respect to $s$, yields:

$$
\tau_{l} \hat{n}=-\hat{\lambda} K_{1} \cos \psi-\left(K_{2}+\psi^{\prime}\right)\left(\hat{\omega}_{1} \sin \psi-\hat{\omega}_{2} \cos \psi\right),
$$

where $\tau_{b}$ is the torsion of union curves and $\hat{n}$ are the components of the principal normal such that $\hat{n}=1 / K_{b}\left(d^{2} x / d s^{2}\right)$.

Multiplying equation (3.11) by $\hat{n}$ and applying equations (2.1), (2.2), (2.4), (3.2a) and (3.2b) we obtain

$$
\begin{aligned}
\tau_{b}=-k_{c} \Pi_{1} \cos \psi-\left(K_{2}+\psi^{\prime}\right) / \Pi_{1} \cdot\left\{\left(\mu_{a \beta} \varrho^{\alpha}+k_{n} \nu_{\beta}\right) u^{\prime \beta} \sin \psi-\right. \\
\left.-e_{a \beta}\left(k_{n} p^{\alpha} \mu_{\gamma}^{\beta}-p^{\alpha} \varrho^{\beta} \nu_{\gamma}+q \varrho^{\beta} \mu_{\gamma}^{\alpha}\right) u^{\beta} \cos \psi\right\},
\end{aligned}
$$

which is the expression for the torsion of union curves.

4. Hyperasymptotic curves. A curve $C$ is said to be a hyperasymptotic curve if the principal normal to the curve $C$ lies in the relative normal plane.

Thus by definition we have for a hyperasymptotic curve

$$
d^{2} \underline{x} / d s^{2} \cdot \hat{\omega}_{1} \times \hat{\omega}_{2}=0 \text {. }
$$

Equation (4.1), in virtue of (2.1) and (3.2b), leads to

$$
p_{\alpha} \varrho^{\alpha}+q k_{n}=0,
$$

which is the equation of hyperasymptotic curves as obtained by Mishra [2].

To obtain an alternative form we express $d^{2} \underline{x} / d s^{2}$ as

$$
d^{2} \underline{x} / d s^{2}=K_{p}\left(\hat{\omega}_{1} \cos \varphi+\hat{\omega}_{2} \sin \varphi\right),
$$

where $K_{p}$ is the curvature of the hyperasymptotic curves and $p$ is the angle between the principal normal to the curve $C$ and $\hat{\omega}_{1}$.

Multiplying equation (4.3) by $\hat{\omega}_{1}$ and $\hat{\omega}_{2}$ respectively and using equations (2.1), (2.2), (2.4), (3.2a) and (3.2b) we obtain after simplification

$$
K_{1} K_{p} \cos \varphi=\left(\mu_{\alpha \gamma} \varrho^{a}+\nu_{\gamma} k_{n}\right) u^{\prime \gamma}
$$

and

$$
K_{1} K_{p} \sin \varphi=e_{a \beta} u^{\prime \delta}\left(\varrho^{a} p^{\beta} v_{\delta}-q \varrho^{\alpha} \mu_{\delta}^{\beta}-k_{n} p^{\beta} \mu_{\delta}^{\alpha}\right)
$$


Squaring equations $(4.4 \mathrm{a}, \mathrm{b})$ and adding we get

$$
\begin{aligned}
\Pi_{1}^{\alpha} \Pi_{p}^{2}=\left\{e_{\alpha \beta} u^{\prime \delta}\left(\varrho^{\alpha} p^{\beta} \nu_{\delta}-q \varrho^{a} \mu_{\delta}^{\beta}-k_{n} p^{\beta} \mu_{\delta}^{a}\right)\right\}^{2} & + \\
& +\left\{\left(\mu_{\alpha \gamma} \varrho^{\alpha}+\nu_{\gamma} k_{n}\right) u^{\prime \gamma}\right\}^{2} .
\end{aligned}
$$

Equations (4.4a, b) and (4.5) are the alternative forms of the curvature and also equation (4.5) is the alternative form of the differential equation of hyperasymptotic curves in the three-dimensional Euclidean space.

Since for an $N_{\lambda}$-curve [7] we have:

$$
\mu_{a \gamma} \varrho^{a}+k_{n} \nu_{y}=0
$$

therefore equation (4.4a) leads to $K_{1} K_{p} \cos q^{\prime}=0$, which implies:

THEOREN (4.1). A necessary and sufficient condition for a hyperasymptotic curve to be a $N_{\lambda}$-curve is that it satisfies one of the following conditions:

(i) the absolute curvature of the congruence vanishes,

(ii) the curvature of the hyperasymptotic curve vanishes,

(iii) the principal normal to the curve $C$ is orthogonal to $\hat{\omega}_{1}$.

Since for a $\beta$-curve $[4]$ we have:

$$
e_{\mathrm{u} \beta} u^{\prime \delta}\left[\varrho^{\alpha} p^{\beta} v_{\delta}-\mu_{\delta}^{\beta}\left(q \varrho^{\alpha}-k_{n} p^{\alpha}\right)\right]=0,
$$

therefore equation (4.4b) leads to $K_{1} K_{p} \sin \varphi=0$, which implies:

Theorem (4.2). A necessary and sufficient condition for a hyperasymptotic curve to be a $\beta$-curve is that it satisfies one of the following conditions :

(i) the absolute curvature of the congruence vanishes,

(ii) the curvature of the hyperasymptotic curve vanishes,

(iii) the principal normal to the curve $O$ is parallel to $\hat{\omega}_{1}$.

Multiplying equation (4.3) by $\hat{N}$ in the sense of inner product and using (2.1), (2.2), (2.4) and (3.2b) we obtain

$$
K_{1} k_{n}=K_{p}\left\{v_{\gamma} u^{\prime \gamma} \cos \varphi+e_{\alpha \beta} p^{a} \mu_{\gamma}^{\beta} u^{\prime \gamma} \sin \varphi\right\} .
$$

Similarly, taking the scalar product of (4.3) bJ $\underline{x}_{, \beta} \varrho^{\beta}$ we get

$$
K_{1} k_{\sigma}^{2}=K_{p}\left\{\mu_{\beta \delta} \varrho^{\beta} u^{\prime \delta} \cos \varphi+e_{\alpha \beta} \varrho^{\alpha} u^{\prime \gamma^{\prime}}\left(p^{\beta} \nu_{y}-q \mu_{\gamma}^{\beta}\right) \sin \varphi\right\} .
$$

Equations (4.8) and (4.9) are the expressions for the curvature of hyperasymptotic curves.

Differentiation of (4.3) with respect to $s$, gives, by (2.4), the following relation:

$$
\begin{aligned}
d^{3} \underline{x} / d s^{3}=-\hat{\lambda} K_{1} \cos \varphi & +\hat{\omega}_{1}\left(K_{p}^{\prime} \cos \varphi-K_{p} \sin \varphi \cdot \varphi^{\prime}-K_{2} \sin \varphi\right)+ \\
& +\hat{\omega}_{2}\left(K_{p}^{\prime} \sin \varphi+K_{p} \cos \varphi \cdot \varphi^{\prime}+K_{2} \cos \varphi\right) .
\end{aligned}
$$


Let $\tau_{p}$ be the torsion of the hyperasymptotic curves; then

$$
\tau_{p}=\frac{1}{K_{p}^{2}}\left(d \underline{x} / d s \cdot d^{2} \underline{x} / d s^{2} \times d^{3} \underline{x} / d s^{3}\right) .
$$

Equation (4.11), in view of $(3.2 \mathrm{a}, \mathrm{b})$ and $(4.10)$, takes the form

$$
\begin{aligned}
\tau_{p}=\frac{1}{K_{p}^{2}}[ & p_{a} u^{\prime \alpha}\left\{\left(K_{2}+\varphi^{\prime} K_{p}\right)\left(K_{p} \cos ^{2} \varphi+\Pi_{p}^{\prime} \sin ^{2} \varphi\right)+\right. \\
& \left.+K_{p}^{\prime}\left(K_{p}-K_{p}^{\prime}\right) \sin \varphi \cos \varphi\right\}-\mu_{a \gamma} u^{\prime a} u^{\prime \gamma} K_{p}^{\prime} \sin \varphi \cos \varphi+ \\
& \left.+K_{p} \cos ^{2} \varphi e_{a \beta}\left(p^{\beta} v_{\delta}-q \mu_{\sigma}^{\beta}\right) u^{\prime \alpha} u^{\prime \sigma}\right] .
\end{aligned}
$$

Thus equation (4.12) represents the torsion of the hyperasymptotic curves in the three-dimensional Euclidean space.

5. Hypernormal curves. A curve $O$ is said to be a hypernormal curve if its tangent vector lies in the relative normal plane.

Thus by definition for a hypernormal curve we have

$$
d \underline{x} / d s \cdot \hat{\omega}_{1} \times \hat{\omega}_{2}=0 .
$$

Equation (5.1), in virtue of (2.1) and (3.2a), leads to

$$
p_{a} u^{\prime a}=0
$$

which is the differential equation of hypernormal curves obtained by Bhattacharya. [1]. However, the word "hypernormal" has been used by latter authors. form:

To obtain an altcrnative form wo express $d x / d s$ in the following

$$
d \underline{x} / d s=\hat{\omega}_{1} \cos \theta+\hat{\omega}_{\mathrm{g}} \sin \theta,
$$

where $\theta$ is the angle between $d \underline{x} / d s$ and $\hat{\omega}_{1}$.

From equation (5.3) we cañ easily obtain

$$
K_{1} \cos \theta=\mu_{a \beta} u^{\prime \alpha} u^{\prime \beta}
$$

and

$$
\pi_{1} \sin \theta=e_{\alpha \beta} u^{\prime a} u i^{\prime \gamma}\left(p^{\beta} \nu_{\gamma}-q \mu_{\gamma}^{\beta}\right) .
$$

Squaring $(5.4 a, b)$ and adding we get

$$
\left(\mu_{a \beta} u u^{\prime \alpha} u^{\prime p}\right)^{2}+\left\{e_{\alpha \beta} u^{\prime \alpha}\left(p^{\beta} \nu_{\gamma}-q \mu_{\gamma}^{\beta}\right) u u^{\prime \gamma}\right\}^{2}=K_{1}^{2} .
$$

Equation (5.5) is the alternative form of the differential equation of hypernormal curves in the three-dimensional Euclidean space. This also gives the absolute curvature of the congruence relative to a hypernormal curve. 
Since for a $T_{\lambda}$-curve [7] we have:

$$
\mu_{\alpha \beta} u^{\prime \alpha} u^{\prime \beta}=0,
$$

therefore equation (5.4a) implies $K_{1} \cos \theta=0$, which leads to

THEOREM (5.1). The necessary and sufficient condition for a hypernormal curve to be a $T_{\lambda}$-curve is that either the tangent to the curve is orthogonal to $\hat{\omega}_{1}$ or the absolute curvature of the congruence vanishes.

The equation of the intersector net of the developables of the congruence is given by Springer [6] in the form

$$
e_{\alpha \beta} u^{\prime \alpha} u^{\prime \gamma}\left(p^{\beta} \nu_{\gamma}-q \mu_{\gamma}^{\beta}\right)=0,
$$

which, when used in (5.4b), implies $K_{1} \sin \theta=0$. Thus:

THEOREM (5.2). A hypernormal curve also satisfies the equation of the intersector net of the developables of the congruence if and only if it satisfies either of the following:

(i) the absolute curvatuve of the congruence vanishcs,

(ii) the tangent vector to the curve $C$ is parallel to $\hat{\omega}_{1}$.

Differentiating equation (5.3) with respect to $s$ we obtain

$$
d^{2} \underline{x} / d s^{2}=-\hat{\lambda} K_{1} \cos \theta-\left(\theta^{\prime}+K_{2}\right)\left(\hat{\omega}_{1} \sin \theta-\hat{\omega}_{2} \cos \theta\right) .
$$

Squaring (5.8) and putting $K_{t}=\left|d^{2} \underline{x} / d s^{2}\right|$ we get

$$
K_{i}^{2}=\left(K_{2}+\theta^{\prime}\right)^{2}+K_{2}^{1} \cos ^{2} \theta,
$$

which gives an expression for the curvature $K_{t}$ of hypernormal curver.

For $\theta=0$, equation (5.9) reduces to

$$
K_{i}^{2}=K_{1}^{2}+K_{2}^{2}
$$

which yields :

THeORen (5.3). If a hypernormal curve $C$, whose tangent is parallel to $\hat{\omega}_{1}$, satisfies any two of the following conditions:

(i) the curvature of the hypernormal curve is zero,

(ii) the absolute curvature of the congruence is zero,

(iii) the absolute torsion of the congruence is zero, then it satisfies also, the third one.

For $\theta=\pi / 2$, equation (5.9) leads to $K_{t}=K_{2}$, which implies:

THEOREMI (5.4). If, for a hypernormal curve its tangent is orthogonal to $\hat{\omega}_{1}$, then its curvature is equal to the absolute torsion of the congruence.

For $\dot{K}_{1}=0$, equation (5.9) implies:

$$
\theta^{\prime}=\left(K_{t}-K_{2}\right)
$$


Thus:

THEOREn (5.5). If, for a hypernormal ourve $C$, the absolute curvature of the congruence vanishes, then the rate of change of the angle between the tangent to the curve $C$ and $\hat{\omega}_{1}$ is the difference of the curvature of the curve and the absolute torsion of the congruence.

From equation (5.8) the normal curvature and geodesic curvature are calculated by multiplying it by $\hat{N}$ and $\underline{x}, \varrho^{\delta}$, respectively, in the sense of inner product in the following form:

$$
K_{1} l_{n}=\left(K_{2}-\theta^{\prime}\right)\left(e_{a \beta} p^{a} \mu_{\gamma}^{\beta} u^{\prime \gamma} \cos \theta-v_{\beta} u^{\prime \beta} \sin \theta\right)-q K_{1} \cos \theta
$$

and

$$
\begin{aligned}
K_{1} k_{o}^{2}=\left(K_{2}+\theta^{\prime}\right)\left\{\mu_{\beta \delta} \varrho^{\delta} u^{\prime \beta} \sin \theta+\left(e_{\delta a} \varrho^{\delta} p^{a} \nu_{\gamma} u^{\prime \gamma}+\right.\right. \\
\left.\left.\quad+q e_{\beta \delta} \mu_{\gamma}^{\beta} u^{\prime \gamma} q \varrho^{\delta}\right) \cos \theta\right\}-\varrho_{a} p^{a} K_{1} \cos \theta .
\end{aligned}
$$

Differentiating equation (5.8) we get after suitable calculations,

$$
\begin{gathered}
d^{3} \underline{x} / d s^{3}=\hat{\lambda}\left\{K_{1}\left(K_{2}+2 \theta^{\prime}\right) \sin \theta-K_{1}^{\prime} \cos \theta\right\}-\hat{\omega}_{1}\left\{\left(\theta^{\prime 2}+K_{1}^{2}+\right.\right. \\
\left.\left.+K_{2}^{2}\right) \cos \theta+\left(K_{2}^{\prime}+\theta^{\prime \prime}\right) \sin \theta\right\}+\hat{\omega}_{2}\left\{\left(K_{2}^{\prime}+\theta^{\prime \prime}\right) \cos \theta-\left(K_{2}+\theta^{\prime}\right)^{2} \sin \theta\right\} .
\end{gathered}
$$

Let $\tau_{t}$ be the torsion of the hypeinormal curve; then we have

$$
\tau_{t}=\frac{1}{\Pi_{i}^{2}}\left(d \underline{x} / d s \cdot d^{2} \underline{x} / d s^{2} \times d^{3} \underline{x} / d s^{3}\right)
$$

which, by (5.3), (5.8) and (5.11), leads after simplification to

$$
\begin{aligned}
\tau_{i}=\frac{1}{K_{t}^{2}} & {\left[\left(K_{2}+\theta^{\prime}\right)\left\{K_{1}\left(K_{2}+2 \theta^{\prime}\right) \sin \theta-K_{1} \cos \theta\right\}+\right.} \\
& +K_{1} \cos \theta \sin \theta\left\{\left(\theta^{\prime 2}+K_{1}^{2}+K_{2}^{2}\right) \cos \theta+\left(K_{2}^{\prime}+\theta^{\prime \prime}\right) \sin \theta\right\}+ \\
& \left.+\Pi_{1} \cos ^{2} \theta\left\{\left(K_{2}^{\prime}+\theta^{\prime \prime}\right) \cos \theta-\left(K_{2}+\theta^{\prime}\right)^{2} \sin \theta\right\}\right]
\end{aligned}
$$

For $\theta=\pi / 2$, equation $(5.16)$ gives $K_{1}=\tau_{t}$, which implies:

THEOREM (5.6). If, for a hypernormal curve its tangent is orthogonal to $\hat{\omega}_{1}$, then its torsion is equal to the absolute curvature of the congruence.

Remark. Some properties of these curves can be studied by taking normal congruence and the congruence tangential to a one parameter family of curves. Also some results may be obtained in relation to other curves.

Acknowledgements. We are grateful to Dr. M.D. Upadhyay for his guidance and help in the preparation of this paper. Our sincere thanks are also due to the referee for his valuable suggestions in the improvement of this paper. The first author (H.K.N. Trivedi) is thankful to U.G.C. India for the award of a Senior Research Followship. 


\section{References}

[1] P. B. Bhattacharya, Gurves on the surface of reference whose normal plane at any point contains the ray of the congruence through that point, Ganita, Vol. 1 (1950), p. 23-29.

[2] R. S. Mishra, A problem in rectilinear congruences using tensor calculus, Bull. Cal. Math. Soc., Vol. 42 (1950), p. 118-122.

[3] K. Nirmala, Properties of the intrinsic derivatives of the first and higher orders of the unit vector tangential to the congruence of eurves in $F_{m}$, relative to a curve in $\nabla_{n 2}$, Tensor (N.S.) Vol. 15, No. 2 (1964), p. 120-127.

[4] S. C. Rastogi and H. K. N. Trivedi, On some curves in an Euclidean space of three dimensions (Under Publication).

[5] C. E. Springer, Enion curves and Enion curvature, Bull. Amer. Math. Soc. 51, No. 10 (1945), p. 686-691.

[6] - Rectilinear congruences whose developables intersect a surface in its line of curvature, ibidem 51 (1945), p. 990-996.

[7] H. K. N. Trivedi and S. C. Rastogi, On some special curves in an Euclidean space of three dimensions (Under Publication).

DEPARTMENT OF MATHEMATICS

LUCKNOW UNIVERSITY

LUCKNOW, INDIA 